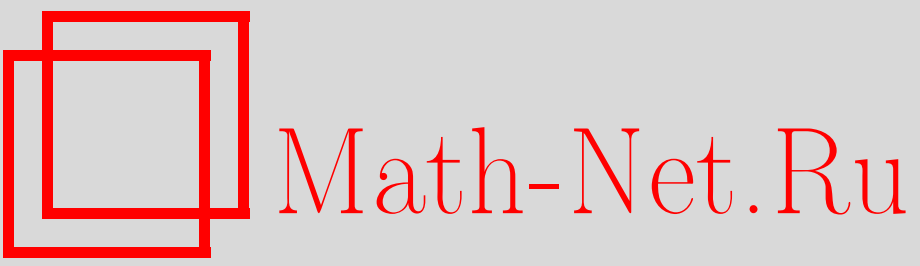

А. В. Киселев, О гамильтоновых потоках на уравнениях Эйлера, ТМФ, 2005, том 144, номер 1, 83-93

DOI: https://doi.org/10.4213/tmf1834

Использование Общероссийского математического портала Math-Net.Ru подразумевает, что вы прочитали и согласны с пользовательским соглашением

http://www.mathnet.ru/rus/agreement

Параметры загрузки:

IP : 54.198 .55 .26

26 апреля 2023 г., 15:56:52 
ТЕОРЕТИЧЕСКАЯ

И МАТЕМАТИЧЕСКАЯ

ФИЗИКА

Том 144, № 1

июль, 2005

(C) 2005 г.

А.В. Киселёв ${ }^{*}$

\section{О ГАМИЛЬТОНОВЫХ ПОТОКАХ НА УРАВНЕНИЯХ ЭЙЛЕРА}

Изучаются свойства потоков гамильтоновых симметрий гиперболических уравнений Эйлера $\mathcal{E}_{\mathrm{EL}}^{\prime}$ лиувиллевского типа. Получено описание нётеровых симметрий, ассоциированных с интегралами данных уравнений. Эти интегралы задают преобразования Миуры из $\mathcal{E}_{\mathrm{EL}}^{\prime}$ в многокомпонентные волновые уравнения $\mathcal{E}$. Используя такие подстановки, удается построить бесконечно-гамильтонову коммутативную подалгебру $\mathfrak{A}$ локальных нётеровых потоков симметрии на $\mathcal{E}$, размножаемых слабо нелокальными операторами рекурсии. Соответствие между схемами Магри для $\mathfrak{A}$ и для индуцированных "модифицированных" гамильтоновых потоков $\mathfrak{B} \subset \operatorname{sym} \mathcal{E}_{\mathrm{EL}}^{\prime}$ таково, что указанные свойства переносятся на $\mathfrak{B}$, а операторы рекурсии для $\mathcal{E}_{\mathrm{EL}}^{\prime}$ факторизуются. Рассмотрены два примера, связанные с двумерной цепочкой Тоды.

Ключевые слова: двумерная цепочка Тоды, уравнение КдФ, уравнение Буссинеска, преобразование Миуры, коммутативные иерархии.

\section{ВВЕ ДЕНИЕ}

В данной работе рассматривается задача построения пар коммутативных иерархий гамильтоновых эволюционных уравнений, связанных преобразованиями Миуры и отождествляемых с подалгебрами Ли алгебр нётеровых симметрий для систем уравнений Эйлера-Лагранжа. K классу гиперболических лагранжевых систем $\mathcal{E}_{\mathrm{EL}}^{\prime}$ лиувиллевского типа применяются две стандартные методики [1]-[3]: подстановки Миуры, заданные интегралами уравнений $\mathcal{E}_{\mathrm{EL}}^{\prime}$, и построение второй гамильтоновой структуры через преобразование Миуры, с помощю которых получено явное описание нётеровых симметрий уравнений $\mathcal{E}_{\mathrm{EL}}^{\prime}$, заданных их интегралами и гамильтоновых относительно (первой) структуры, определяемой из лагранжианов. Также в работе изучаются свойства схем Магри для следующей пары иерархий, связанных подстановкой Миуры: $\mathfrak{A}$, образуюшие которой суть симметрии волнового уравнения, и $\mathfrak{B} \subset \operatorname{sym} \mathcal{E}_{\mathrm{EL}}^{\prime}$. Приведены два примера. В разделе 2 уравнение Кортевега-де Фриза (КдФ)

$$
s_{t_{1}}=-\beta s_{x x x}+\frac{3}{2} s_{x}^{2}, \quad w_{t_{1}}=-\beta w_{x x x}+3 w w_{x}, \quad w=s_{x}, \quad \beta=\mathrm{const},
$$

*Department of Mathematics, Brock University, 500 Glenridge Ave., St. Catharines, Ontario, Canada L2S 3A1; Ивановский государственный энергетический университет, каф.. высшей математики, 153003 Россия, Иваново, ул. Рабфаковская, 34. E-mail: arthemy@poincare.unile.it 
и многокомпонентные модифицированные уравнения КдФ [4] связаны, соответственно, с волновым уравнением и двумерной цепочкой Тоды (в частности, ассоциированной с полупростой алгеброй Ли [5]); построена факторизация оператора рекурсии для уравнения (1) в произведение двух векторнозначных операторов. В разделе 3 показано, что уравнение Буссинеска [6]

$$
\left\{\begin{array} { l } 
{ u _ { t _ { 1 } } = \frac { 1 } { 3 } v _ { x x x } + \frac { 4 } { 3 } v _ { x } ^ { 2 } , } \\
{ v _ { t _ { 1 } } = u _ { x } , }
\end{array} \quad \left\{\begin{array} { l } 
{ U _ { t _ { 1 } } = V _ { x } , } \\
{ V _ { t _ { 1 } } = \frac { 1 } { 3 } U _ { x x x } + \frac { 8 } { 3 } U U _ { x } , }
\end{array} \quad \left\{\begin{array}{l}
U=v_{x}, \\
V=u_{x},
\end{array}\right.\right.\right.
$$

и модифицированное уравнение Буссинеска [7], равно как и гамильтоновы структуры, задающие их коммутативные локальные иерархии, определяются геометрией двухкомпонентного волнового уравнения и двумерной цепочки Тоды, соответственно.

Взаимосвязь между гамильтоновым и лагранжевым подходами к интегрируемым эволюционным уравнениям обсуждалась в работе [8]. В указанной работе представления уравнений в лагранжевой форме получались путем применения преобразования Лежандра к последовательности гамильтонианов исходного эволюционного уравнения. Таким образом, используемый подход был замкнут относительно эволюционных систем и преобразований Миуры между ними вне зависимости от способа их представления. Рассматриваемый ниже подход противоположен подходу работы [8] и основан на интерпретации (би)гамильтоновых иерархий эволюционных уравнений как потоков, заданных подалгебрами алгебры нётеровых симметрий объемлющих систем уравнений Эйлера-Лагранжа (см. также [6]). Используя канонические переменные для лагранжевых систем и трактуя дифференциальную связь между координатами $u$ и импульсами $\mathfrak{m}$ как правило, задаюшее потенциалы Клебша, мы устанавливаем соотношение между потенциальными и непотенциальными компонентами иерархий, которые описывают эволюцию координат и импульсов, соответственно. Сопряженная линеаризация $\left(\ell_{\mathfrak{m}}^{u}\right)^{*}$ указанной выше дифференциальной связи задает первые гамильтоновы структуры для этих иерархий, и в результате их схемы Магри оказываются согласованными. Вторые гамильтоновы структуры задают операторы рекурсии и для иерархий эволюционных уравнений, и для объемлющих их систем Эйлера.

Структура работы такова. В разделе 1 указьвается взаимосвязь между дифференциальным соотношением на координаты и импульсы гиперболических лагранжевых уравнений и гамильтоновыми операторами для алгебры их симметрий. Далее рассматриваются подстановки из лагранжевых уравнений $\mathcal{E}_{\mathrm{EL}}^{\prime}$ лиувиллевского типа, заданные набором их интегралов; в этом случае подстановка Миуры в подалгебру $\mathfrak{A}$ нётеровых симметрий лагранжева волнового уравнения $\mathcal{E}$ задает вторую гамильтонову структуру на $\mathfrak{A}$. Так удается получить пару $\mathfrak{A}, \mathfrak{B}$ последовательностей гамильтоновых потоков на уравнениях $\mathcal{E}, \mathcal{E}_{\mathrm{EL}}^{\prime}$, соответственно, скоррелированных посредством преобразования Миуры. Затем исследуются свойства потоков, содержашихся в $\mathfrak{A}$ и $\mathfrak{B}$, и операторов рекурсии для них; выясняется, какие из полученных свойств иерархии $\mathfrak{A}$, таких как локальность, коммутативность, бигамильтоновость и т.п., имеют место также и для $\mathfrak{B}$.

В разделе 2 приведены необходимые сведения о геометрии двумерной цепочки Тоды $u_{x y}=\exp (K u)$; определения и обозначения следуют работе [9]. Затем рассматривают- 
ся иерархия уравнения КдФ (1) и последовательность многокомпонентных аналогов модифицированного уравнения Кд $\Phi$ [4], связанных с двумерной цепочкой Тоды. Получено разложение оператора рекурсии для уравнения КдФ в произведение векторнозначных операторов; доказывается, что высшие потоки многокомпонентных модифицированных уравнений КдФ локальны и попарно коммутируют.

В разделе 3 показано, что иерархия $\mathfrak{B}$ высших потоков модифицированного уравнения Буссинеска (8) образует коммутативную подалгебру нётеровых симметрий двумерной цепочки Тоды $(9)$, ассоциированной с алгеброй $\mathfrak{s l}_{3}(\mathbb{C})$. Установлено, что интегралы $(7)$ системы (9) задают вторую гамильтонову структуру (10) для уравнения (2); получена факторизация оператора рекурсии для цепочки Тоды.

\section{1. ИЕРАРХИИ И ПРЕОБРАЗОВАНИЯ МИУРЫ}

Рассмотрим лагранжиан первого порядка $\mathcal{L}=\left[L\left(u, u_{x}, u_{y} ; x, y\right) d x \wedge d y\right]$ с плотностью $L=-\sum_{i, j} \bar{\kappa}_{i j} u_{x}^{i} u_{y}^{j} / 2+H(u ; x, y)$, где $\bar{\kappa}$ - невырожденная вещественная симметричная постоянная $(r \times r)$-матрица. Выберем в качестве "времени" координату $y$, оставив $x$ пространственной переменной, и обозначим через $\mathfrak{m}_{j}=\partial L / \partial u_{y}^{j}$ координату, сопряженную к $j$-й зависимой переменной $u^{j}$ (j-й импульс) при $1 \leqslant j \leqslant r$,

$$
\mathfrak{m}=-\frac{1}{2}\left(\bar{\kappa} u_{x}\right)^{*}
$$

Ниже будет показано, что сопряженная обратная линеаризация $D_{x}^{-1} \circ \bar{\kappa}^{-1}$ дифференциальной связи (3) между координатами и импульсами является гамильтоновой структурой для гиперболического уравнения Эйлера-Лагранжа $\mathcal{E}_{\mathrm{EL}}=\left\{\mathbf{E}_{u}(\mathcal{L})=0\right\}$ и алгебры sym $\mathcal{L}$ нётеровых симметрий этого уравнения. Применяя преобразование Лежандра $H d x \wedge d y=\left\langle\mathfrak{m}, \partial L / \partial u_{y}\right\rangle-\mathcal{L}$, поставим в соответствие лагранжиану $\mathcal{L}$ гамильтониан $\mathcal{H}(u, \mathfrak{m})=[H d x]$. Гиперболическое лагранжево уравнение $\mathcal{E}_{\mathrm{EL}}$ эквивалентно системе

$$
u_{y}=\mathbf{1} \cdot \mathbf{E}_{\mathfrak{m}}(\mathcal{H}), \quad \mathfrak{m}_{y}=-\mathbf{1} \cdot \mathbf{E}_{u}(\mathcal{H})
$$

которая определена канонической гамильтоновой структурой $\left(\begin{array}{cc}0 & \mathbf{1} \\ -1 & 0\end{array}\right)$. В силу соотношений

$$
\frac{1}{2} \mathbf{E}_{\mathfrak{m}}=\bar{\kappa}^{-1} \cdot D_{x}^{-1} \cdot \mathbf{E}_{u}, \quad \mathbf{E}_{u}=\frac{1}{2} D_{x} \circ \bar{\kappa} \cdot \mathbf{E}_{\mathfrak{m}},
$$

уравнения движения разделяются и принимают эволюционную форму

$$
u_{y}=A_{1} \circ \mathbf{E}_{u}([H[u] d x]), \quad \mathfrak{m}_{y}=-\frac{1}{2} \hat{A}_{1} \circ \mathbf{E}_{\mathfrak{m}}([H[\mathfrak{m}] d x])
$$

таким образом, теперь они заданы парой взаимно обратных гамильтоновых операторов $A_{1}=\bar{\kappa}^{-1} \cdot D_{x}^{-1}$ и $\hat{A}_{1}=D_{x} \cdot \bar{\kappa}$.

Пусть гиперболическое лагранжево уравнение $\mathcal{E}_{\mathrm{EL}}$ допускает поток симметрии $u_{t}=$ $\phi\left(u_{x}, u_{x x}, \ldots\right) \equiv \phi\left[u_{x}\right]$, где $t$ - параметр вдоль интегральных траекторий. Тогда эволюция $\mathfrak{m}_{t}$ импульсов задается непотенциальным уравнением $\mathfrak{m}_{t}=-(\bar{\kappa} / 2) \cdot D_{x}(\phi[\mathfrak{m}])$; уравнение (1) поставляет пример потоков симметрии волнового уравнения $s_{x y}=0$. 
ЛЕмма 1 [10]. Пусть $\mathcal{E}=\left\{\boldsymbol{u}_{x y}=\boldsymbol{f}(\boldsymbol{u} ; x, y)\right\}$ - система квазилинейных гиперболических уравнений, допускающая закон сохранения

$$
\mathcal{H}=H d x+\cdots: \quad d_{h}(\mathcal{H})=\nabla\left(\boldsymbol{u}_{x y}-\boldsymbol{f}\right) .
$$

Тогда производящее сечение $\psi \equiv \nabla^{*}(1)$ для $\mathcal{H}$ имеет вид $\psi=-D_{x}\left(\mathbf{E}_{\boldsymbol{u}}(H d x)\right)$.

По теореме Нётер [11] нётеровы симметрии самосопряженных лагранжевых систем $\mathcal{E}_{\mathrm{EL}}=\left\{\mathbf{E}_{u}(\mathcal{L})=0\right\}$ совпадают с производящими сечениями их законов сохранения; согласно [12] корреляция между нётеровыми симметриями $\varphi_{\mathcal{L}}$ и производяшими сечениями $\psi$ для несамосопряженных лагранжевых уравнений $\mathcal{E}_{\mathrm{EL}} \simeq\left\{\bar{\kappa}^{-1} \cdot \mathbf{E}_{u}(\mathcal{L})=0\right\}$ с равным единичной матрице символом такова: $\psi=\bar{\kappa} \varphi_{\mathcal{L}}$.

Пусть $\phi$ - нётерова симметрия гиперболической лагранжевой системы, тогда $u_{t}=$ $\mathbf{E}_{\mathfrak{m}}(\mathcal{H}) / 2, \mathfrak{m}_{t}=-\mathbf{E}_{u}(\mathcal{H}) / 2$, где $\mathcal{H}=[H d x]$ и $H$ - сохраняюшаяся плотность, откуда $u_{t}=\bar{\kappa}^{-1} \cdot D_{x}^{-1} \mathbf{E}_{u}(\mathcal{H}), \mathfrak{m}_{t}=-D_{x} \cdot \bar{\kappa} \mathbf{E}_{\mathfrak{m}}(\mathcal{H}) / 2$. Таким образом, оба уравнения $u_{t}=\phi$ и $\mathfrak{m}_{t}=-\bar{\kappa} D_{x}(\phi) / 2$ гамильтоновы одновременно, и их гамильтоновы операторы $A_{1}$ и $\hat{A}_{1}$ взаимно обратны (см., например, [13]). Индуцированную эволюцию импульсов $\mathfrak{m}$ можно получить двумя способами: варьируя гамильтониан, при этом $\mathfrak{m}_{t}=-\mathbf{E}_{u}(\mathcal{H})=$ $-\left(\ell_{\mathfrak{m}}^{u}\right) *\left(\mathbf{E}_{\mathfrak{m}}(\mathcal{H})\right)$, или явно используя соотношение $(3)$, при этом $\mathfrak{m}_{t}=\ell_{\mathfrak{m}}^{u}\left(\mathbf{E}_{\mathfrak{m}}(\mathcal{H})\right)$. Для гиперболических лагранжевых уравнений результаты согласованы, поскольку вьполнено условие $\left(\ell_{\mathfrak{m}}^{u}\right)^{*}=-\ell_{\mathfrak{m}}^{u}$. Далее, пусть $R$ - оператор рекурсии для гиперболического лагранжева уравнения $\mathcal{E}_{\mathrm{EL}}=\left\{\mathbf{E}_{u}(\mathcal{L})=0\right\}$, порождаюший из некоторого потока $\phi_{-1}$ коммутативную цепочку $u_{t_{i}}=\phi_{i}\left[u_{x}\right]$ гамильтоновых симметрий. Понятно, что рекурсия $R$ оказывается общей для всех уравнений $u_{t_{i}}=\phi_{i}$, а оператор $R^{*}$ последовательно отображает скорости $\mathfrak{m}_{t}$ эволюции импульсов (см. работу [14]).

В дальнейшем мы будем рассматривать класс гиперболических лагранжевых уравнений лиувиллевского типа [1], [5], [15], допускающих нетривиальные интегралы, т.е. функционалы $w \in \operatorname{ker} \bar{D}_{y}$, лежашие в ядре полной производной $D_{y}$, ограниченной на рассматриваемое уравнение $\mathcal{E}_{\mathrm{EL}}^{\prime}$. Приведенные ниже рассуждения справедливы с точностью до перестановки $x \leftrightarrow y$ в том случае, если она является симметрией уравнения $\mathcal{E}_{\mathrm{EL}}^{\prime}$, в противном случае замена $x$ на $y$ (и наоборот) поставляет "зеркальные копии" всех конструкций и их свойств.

Если интегралы $w[\overline{\mathfrak{m}}]$ зависят от импульсов $\overline{\mathfrak{m}}$ лагранжева уравнения лиувиллевского типа, то само оно и его симметрии отображаются преобразованием Миуры $\mathfrak{m}=w[\overline{\mathfrak{m}}]$ в многокомпонентное волновое уравнение $\mathcal{E}$ и, соответственно, в его алгебру симметрий; при этом количество компонент в волновом уравнении равно числу интегралов $w$, задающих преобразование Миуры. В рассматриваемой ситуации определены еще два преобразования: ковариантное отображение $w_{*}: \operatorname{sym} \mathcal{E}_{\mathrm{EL}}^{\prime} \rightarrow \operatorname{sym} \mathcal{E}$ потоков симметрии (см. утверждение 4 в работе [1]) и действуюшее контравариантно отображение $w^{*}$ гамильтонианов $H d x$ (а значит, и гамильтоновых потоков). Действительно, в силу равенства $\mathfrak{m}=w[\overline{\mathfrak{m}}]$ вариационные производные относительно импульсов $\mathfrak{m}$ и модифицированных импульсов $\overline{\mathfrak{m}}$ связаны соотношением $\mathbf{E}_{\overline{\mathfrak{m}}}=\square \circ \mathbf{E}_{\mathfrak{m}}$, где $\square=\left(\ell_{\mathfrak{m}}^{\overline{\mathfrak{m}}}\right)^{*}$. Используя лемму 1 , получим 
УТВЕРЖДЕНИЕ 1. Присоединенная линеаризация $\square=\left(\ell_{w}^{\overline{\mathrm{m}}}\right)^{*}$ интегралов $w$ лагранжевых уравнений $\mathcal{E}_{\mathrm{EL}}^{\prime}=\left\{\mathbf{E}_{\bar{u}}\left(\mathcal{L}^{\prime}\right)=0\right\}$ лиувиллевского типа, вычисленная относительно импульсов $\overline{\mathfrak{m}}$, факторизует нётеровы симметрии $\varphi_{\mathcal{L}^{\prime}}: \varphi_{\mathcal{L}^{\prime}}=$ $\square\left(\mathbf{E}_{\mathfrak{m}}(\mathcal{H}[\mathfrak{m}])\right)$, причем плотность гамильтониана $\mathcal{H}$ произвольна.

Примеры, иллюстрируюшие утверждение 1 , можно найти в [4], [12]; взаимосвязь структуры образующих алгебры симметрий гиперболических уравнений лиувиллевского типа и их интегралов была исследована в недавней работе [16].

Рассмотрим подстановку $\mathfrak{m}=w[\overline{\mathfrak{m}}]$ из лагранжева лиувиллевского уравнения $\mathcal{E}_{\mathrm{EL}}^{\prime}$ в многокомпонентное волновое уравнение $\mathcal{E}$. Пара отображений $\left(w^{*}, w_{*}\right)$ индуцирует вторую гамильтонову структуру $\hat{A}_{2}$ на нётеровых симметриях уравнения $\mathcal{E}$ [3]. Именно, пусть задана последовательность $\mathfrak{A}$ нётеровых потоков на $\mathcal{E}$, соответствуюших сохраняюшимся плотностям $H_{i}[\mathfrak{m}]$ (плотностям гамильтонианов) относительно первых структур $\left(\left(\ell_{\mathfrak{m}}^{u}\right)^{*}\right)^{ \pm 1}$. Тогда по каждому потоку $\phi_{i-1}=\mathbf{E}_{\mathfrak{m}}\left(\mathcal{H}_{i}\right)$ на уравнении $\mathcal{E}$ можно построить поток $\bar{u}_{t_{i}}=\varphi_{i}=\square\left(\phi_{i-1}\right)$ на $\mathcal{E}_{\mathrm{EL}}^{\prime}$. Воспользуемся условием $\left(\ell_{\mathfrak{m}}^{u}\right)^{*}=-\ell_{\mathfrak{m}}^{u}$. Итак, корректно определена эволюция модифишированных импульсов $\overline{\mathfrak{m}}$. Эволюция $⿹_{\square\left(\phi_{i-1}\right)}(w)$ подстановки $w$ вдоль потока $\varphi_{i} \in \mathfrak{B}$ согласована с потоком $\phi_{i}$, следуюшим за $\phi_{i-1}$ в $\mathfrak{A}$, если вторая гамильтонова структура $\hat{A}_{2}$ для $\mathfrak{A}$ удовлетворяет операторному уравнению

$$
\ell_{\mathfrak{m}}^{\overline{\mathfrak{m}}} \circ\left(\ell_{\frac{\bar{u}}{\mathfrak{m}}}\right)^{*} \circ\left(\ell_{\mathfrak{m}}^{\overline{\mathfrak{m}}}\right)^{*}=\hat{A}_{2},
$$

и наоборот, оператор $\hat{A}_{2}$, заданный этим уравнением (4), всегда является гамильтоновой структурой для $\mathfrak{A}$. Иными словами, уравнение (4) - это условие приводимости структуры $\hat{A}_{2}$ к канонической форме " $d / d x$ ", которое ограничивает набор допустимых преобразований Миуры $w=w[\overline{\mathfrak{m}}]$ и модифицированных уравнений Эйлера $\mathcal{E}_{\mathrm{EL}}^{\prime}$ при фиксированном операторе $\hat{A}_{2}$.

ЗАмЕчАниЕ 1 . Если окажется, что операторы $\hat{A}_{1}$ и $\hat{A}_{2}$ совместны, а потому последовательность $\mathfrak{A}$ коммутативна и оператор рекурсии $R=\left(\hat{A}_{2} \circ \hat{A}_{1}^{-1}\right)^{*}$ для уравнения $\mathcal{E}$ яВляется также и рекурсией для $\mathfrak{A}$, то бигамильтонова иерархия $\mathfrak{A}$ допускает бесконечный набор гамильтоновых структур. Действительно, пусть $\varepsilon=\left\{\boldsymbol{u}_{t}=\hat{A}_{1}(\psi[\boldsymbol{u}])\right\}$ есть некоторое гамильтоново уравнение, допускаюшее рекурсию $R$ (в обсуждаемом вьше случае оператор $R$ размножает коммутируюшие потоки симметрий волнового уравнения $\mathcal{E}$ ). Тогда уравнение $\varepsilon$ гамильтоново также и относительно операторов $\hat{A}_{i}=R^{i-1} \hat{A}_{1}$ при условии, что они кососопряжены (что выполнено в силу (4)). Доказательство этого свойства основано на следуюшем рассуждении. Согласно [14], [17] гамильтоновы операторы $A$ для $\varepsilon$ удовлетворяют соотношению $\ell_{\varepsilon} A+A \ell_{\varepsilon}^{*}=0$, причем обратное также верно при дополнительных предположениях регулярности. Поскольку $R$ является рекурсией для $\varepsilon$, то выполнено $\ell_{\varepsilon} R=R \ell_{\varepsilon}$. В самом деле, сушествует такой оператор $\bar{R}$, что выполнено $\ell_{\varepsilon} R=\bar{R} \ell_{\varepsilon}$. Прокоммутируем это равенство с оператором умножения на $t$ и получим $\bar{R}=R$. Следовательно, имеет место соотношение $\ell_{\varepsilon}(R A)+(R A) \ell_{\varepsilon}^{*}=0$, и поэтому оператор $R A$ также гамильтонов. Теперь повторим рассуждения по индукции необходимое число раз. Приведенное выше обоснование есть строгое доказательство для тех операторов $\hat{A}_{i}$, которые являются дифференциальными (т.е. локальными по $D_{x}$ ), в 
противном случае все соотношения следует трактовать формально, применяя их к набору переменных, пополненному нелокальностями $D_{x}^{-1}(\boldsymbol{u})$. Получаемые операторы $\hat{A}_{i}$ не обязательно совместны; отметим также, что, используя пару локальных дифференциальных операторов, можно, вообе говоря, получить нелокальную последовательность $\mathfrak{A}$. Ниже мы обсудим свойства локальности более подробно.

Рассмотрим последовательность $\mathfrak{B} \subset \operatorname{sym} \mathcal{E}_{\mathrm{EL}}^{\prime}$ нётеровых симметрий $\varphi_{i}$, заданных оператором рекурсии $R^{\prime}=\square \circ A_{1} \circ \square^{*} \circ \ell \frac{\bar{u}}{\mathfrak{m}}$ и согласованных с бигамильтоновой последовательностью нётеровых симметрий многокомпонентного волнового уравнения $\mathcal{E}$ посредством преобразования Миуры $\mathfrak{B} \rightarrow \mathfrak{A}$; это преобразование задано интегралами $w$. Последовательности $\mathfrak{A}$ и $\mathfrak{B}$ допускают обший набор гамильтонианов и, по построению, вторая гамильтонова структура $\hat{A}_{2}$ для $\mathfrak{A}$ индуцирована первой гамильтоновой структурой $\widehat{B}_{1}=\left(\ell \frac{\bar{u}}{\mathfrak{m}}\right)^{*}$ для $\mathfrak{B}$, что отображено на следующей диаграмме [2]:

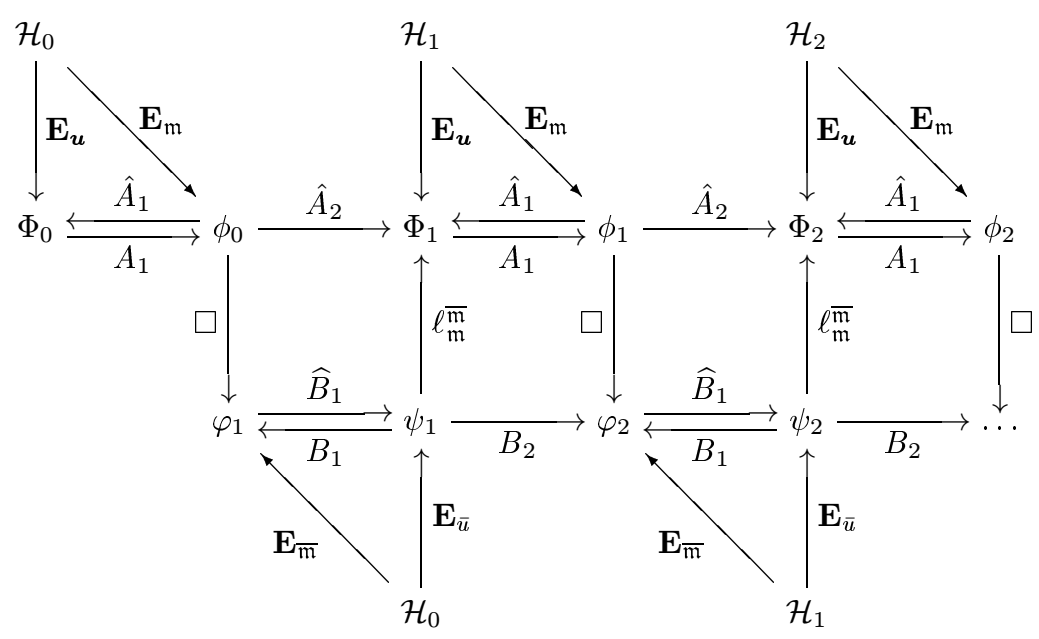

И схемы Магри [2] для $\mathfrak{A}$ и $\mathfrak{B}$, и факторизация (4) оператора $\hat{A}_{2}$ (см. [3]) стандартны. Однако теперь понятно, что nepвые гамильтоновы структуры $\hat{A}_{1}$ и $\widehat{B}_{1}$ заданы лагранжианами объемлющих уравнений Эйлера, а оператор $\square$ определяется по интегралам систем $\mathcal{E}_{\mathrm{EL}}^{\prime}$ лиувиллевского типа.

Если гамильтоновы операторы $\hat{A}_{1}=\left(\ell_{\mathfrak{m}}^{u}\right)^{*}$ и $\hat{A}_{2}$ совместны, то функционалы $\mathcal{H}_{i}[\mathfrak{m}]$ находятся в инволюции относительно обеих структур, и потоки $\phi_{i}$ коммутируют [2]; согласно замечанию 1 иерархия $\mathfrak{A}$ допускает бесконечный набор гамильтоновых операторов. Кроме того, имеем

УТВЕРЖДЕНИЕ 2. Пусть оператор рекурсии $R$ для эволючионного уравнения $\varepsilon=$ $\left\{\boldsymbol{u}_{t}=\phi[\boldsymbol{u}]\right\}$ построен по набору абелевых нелокальных переменных (выражсений, нелокальных относительно всех входящих в в переменных и заданных сохраняющимися токами $\eta_{\alpha}$ для $\varepsilon$, см. [9]). Тогда $R$ слабо нелокален: $R=$ дифференциальная часть $+\sum_{\alpha} \varphi_{\alpha} D_{x}^{-1} \circ \psi_{\alpha}$, әде $\varphi_{\alpha} \in \operatorname{sym} \varepsilon, \psi_{\alpha}-$ производящее сечение закона сохранения $\eta_{\alpha}$ при всяком $\alpha$. 
Доказательство утверждения 2 следует из определений, и его несложно обобшить на случай нескольких слоев абелевых нелокальных переменных, заданных (нелокальными) законами сохранения. Из сформулированного вьше утверждения следует, что рекурсия $R^{*}: \Phi_{i} \mapsto \Phi_{i+1}$ для иерархии $\mathfrak{A}$ слабо нелокальна в силу разложений $R^{*}=\hat{A}_{2}$ 。 $\hat{A}_{1}^{-1}$ и уравнения (4).

УТВЕРЖДЕНИЕ 3. Если операторы $\hat{A}_{1}$ и $\hat{A}_{2}$ совместны, то потоки $\phi_{i} \in \operatorname{sym} \mathcal{E}$ являются нётеровыми.

Действительно, гомологическая формулировка (см., например, [14]) схемы Магри и тривиальность пуассоновых когомологий относительно структуры $\hat{A}_{1}[18]$ гарантируют существование законов сохранения $\mathcal{H}_{i}$ для $\mathcal{E}$. Далее следует воспользоваться леммой 1 и применить теорему Нётер [11], [12]. Мы также можем сделать вывод о том, что $\mathfrak{A}$ локальна по $w$.

Выясним, какие из перечисленных выше свойств переносятся также и на последовательность $\mathfrak{B}$. Очевидно, $\mathfrak{A}$ и $\mathfrak{B}$ локальны одновременно. Ограничения отображений $B_{2}: \psi_{i} \mapsto \varphi_{i+1}$ и $\widehat{B}_{2}: \varphi_{i} \mapsto \psi_{i+1}$ на функционалы $H[w] d x$, зависяшие от модифицированных переменных $\bar{u}$ лиш через подстановку $w=w[\overline{\mathfrak{m}}]$, являются гамильтоновыми операторами, и функционалы $\mathcal{H}_{i}[w[\overline{\mathfrak{m}}]]$ находятся в инволюции относительно $\widehat{B}_{2}$, поскольку это было верно для $\hat{A}_{3}$. Из замечания 1 понятно, почему тождество Якоби выполняется для операторов $B_{2}, \widehat{B}_{2}$ даже в том случае, когда гамильтонианы $\mathcal{H}$ зависят от $\bar{u}$ произвольным образом.

УТВЕРЖДЕНИЕ 4. В приведенных выше обозначениях и предположениях верно следующее: если потоки $\phi_{i} \in \mathfrak{A}$ коммутируют $u$ ker $\hat{A}_{2}=0$, то последовательность $\mathfrak{B}$ также коммутативна.

ДокАЗАТЕльСтво. Согласно утверждению 1 всякая нётерова симметрия гиперболического лагранжева уравнения $\mathcal{E}_{\mathrm{EL}}^{\prime}$ лиувиллевского типа делится на оператор $\square$ (с точностью до замены $x \leftrightarrow y$, если она является симметрией уравнения $\left.\mathcal{E}_{\mathrm{EL}}^{\prime}\right)$. Коммутатор двух нётеровых симметрий $\square\left(\phi^{\prime}\right), \square\left(\phi^{\prime \prime}\right)$ - вновь нётерова симметрия: $\left\{\square\left(\phi^{\prime}\right)\right.$, $\left.\square\left(\phi^{\prime \prime}\right)\right\}=\square\left(\phi_{\{1,2\}}\right)$, причем выражение $\phi_{\{1,2\}}$ может содержать слагаемые, нетипичные для стандартной скобки Якоби сечений $\phi^{\prime}$ и $\phi^{\prime \prime}$, см. приведенную ниже формулу (6). Из условия (4) следует, что $Э_{\square\left(\phi_{\{1,2\}}\right)}(w)=\hat{A}_{2}\left(\phi_{\{1,2\}}\right)$. Таким образом, если оператор $\hat{A}_{2}$ инъективный, иерархия $\mathfrak{A}$ коммутативна, а последовательность $\mathfrak{B}$ согласована с $\mathfrak{A}$ посредством (4) и (5), то $\mathfrak{B}$ коммутативна.

Из утверждения 4 следует, что рекурсия $R^{\prime}$ для системы $\mathcal{E}_{\mathrm{EL}}^{\prime}$ является обшей для всех уравнений, входяших в $\mathfrak{B}$. Из утверждения 2 мы также заключаем, что оператор рекурсии $\left(R^{\prime}\right)^{*}$, размножаюший непотенциальную компоненту $\mathfrak{B}$, слабо нелокален.

В разделах 2 и 3 приведенная выше схема применена к иерархиям уравнений $\mathrm{Kд} \Phi$ и Буссинеска. Сначала для каждой из них восстанавливается потенциальная компонента $\mathfrak{A}$, для чего первая гамильтонова структура $\hat{A}_{1}$ трактуется как сопряженная линеаризация связи (3), и указываются лагранжианы объемлющих гиперболических уравнений Эйлера-Лагранжа (для чего следует отыскать матрицы $\bar{\kappa}$, поскольку плотности гамильтонианов тривиальны). Затем мы находим такие лагранжевы уравнения $\mathcal{E}_{\mathrm{EL}}^{\prime}$ 
лиувиллевского типа, что соответствующие модифицированные иерархии $\mathfrak{B}$ состоят из потоков их симметрий, а преобразования Миуры $\mathcal{E}_{\mathrm{EL}}^{\prime} \mapsto \mathcal{E}$, переводящие $\mathfrak{B}$ в $\mathfrak{A}$, заданы интегралами уравнений $\mathcal{E}_{\mathrm{EL}}^{\prime}$. Далее, коммутативность потоков $\mathfrak{A}$ переносится на модифицированные потоки (локальные нётеровы симметрии уравнения $\mathcal{E}_{\mathrm{EL}}^{\prime}$ ) и строятся разложения слабо нелокальных операторов рекурсии.

\section{2. ДВУМЕРНАЯ ЦЕПОЧКА ТОДЫ И ИЕРАРХИЯ КДФ}

Начнем с формулировки необходимых свойств двумерной цепочки Тоды. Пусть $r \geqslant 1$, $K=\left\|k_{i j}\right\|$ - произвольная постоянная вешественная невырожденная $(r \times r)$-матрица, а вектор $\vec{a}={ }^{t}\left(a_{1}, \ldots, a_{r}\right)$ таков, что $a_{i} \neq 0$ при всех $1 \leqslant i \leqslant r$ и условие $\kappa_{i j} \equiv a_{i} k_{i j}=\kappa_{j i}$ выполнено для всех элементов матрицы $\kappa=\left\|\kappa_{i j}\right\|$. Тогда матрица $K$ называется $с и м-$ метризуемой; обозначим обратную к ней через $K^{-1}=\left\|k^{i j}\right\|$. Двумерная $r$-компонентная цепочка Тоды, ассоциированная с невырожденной симметризуемой матрицей $K$, имеет вид $\mathcal{E}_{\text {Toda }}=\left\{u_{x y}=\exp (K u)\right\}$. Плотность лагранжиана $\mathcal{L}_{\text {Toda }}$ есть $L_{\text {Toda }}=$ $-\left\langle u_{y}, \kappa u_{x}\right\rangle / 2-\langle\vec{a}, \exp (K u)\rangle$. Обозначим через $\vartheta=\kappa u_{x}$ импульсы, полученные из равенства $(3)$. Компонента $w=\left\langle u_{x}, \kappa u_{x}\right\rangle / 2-\left\langle\vec{a}, u_{x x}\right\rangle$ тензора энергии-импульса для уравне-

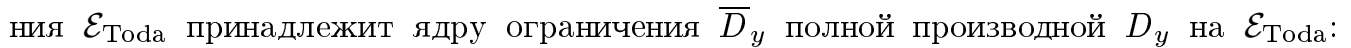
$\bar{D}_{y}(w)=0$. Конформные веса $\vec{\Delta}={ }^{t}\left(\Delta^{1}, \ldots, \Delta^{r}\right)$ полей $\exp (u)$ таковы: $\Delta^{i}=\sum_{j=1}^{r} k^{i j}$. Рассмотрим случай, когда функционал $w$ и его дифференциальные следствия $\boldsymbol{W}$ - единственные решения уравнения $\bar{D}_{y}(\Omega)=0$, зависящие от производных $u$; тогда положим по определению, что матрица $K$ общего положения.

Нётеровы симметрии уравнения $\mathcal{E}_{\text {Тоda }}$, ассоциированного с симметризуемой $(r \times r)$ матрицей $K$ обшего положения, суть $\varphi=\square\left(\mathbf{E}_{w}(H(x, \boldsymbol{W}) d x)\right)$ с точностью до преобразования $x \leftrightarrow y$ (см. [12], а также [10]); в приведенной выше формуле $\square=\left(\ell_{w}^{\vartheta}\right)^{*}=u_{x}+$ $\vec{\Delta} \bar{D}_{x}$ - это векторнозначный дифференциальный оператор первого порядка, а $H$ - произвольная гладкая функция. Оператор $R_{\text {Toda }}=\square \circ \ell_{s}$, где $s_{x}=w$ и $s_{x y}=0$, есть нелокальный оператор рекурсии для алгебры sym $\mathcal{E}_{\text {Toda }}[12]$.

Положим $\phi_{-1}=1$ и зададим три последовательности симметрий при $i \geqslant 0: \varphi_{i}=$ $\square\left(\phi_{i-1}\right) \in \operatorname{sym} \mathcal{E}_{\text {Toda }}, \phi_{i}=R_{\mathrm{pKdV}}\left(\phi_{i-1}\right) \in \operatorname{sym} \mathcal{E}_{\mathrm{pKdV}}$ и $\Phi_{i}=\vartheta_{\phi_{i}}(w) \in \operatorname{sym} \mathcal{E}_{\mathrm{KdV}}$, где уравнения КдФ имеют вид (1) и $\beta \equiv\langle\vec{a}, \vec{\Delta}\rangle$. Скорости эволюции $\Phi_{i}$ и $\phi_{i}$ являются элементами локальных коммутативных бигамильтоновых иерархий потенциальных уравнений Кд $\Phi w_{t_{1}}=\Phi_{1}$ и $s_{t_{1}}=\phi_{1}$, соответственно. Уравнение $u_{t_{1}}=\varphi_{1}=\square\left(\phi_{0}\right)-$ это $r$-компонентный аналог потенциального модифицированного уравнения КдФ и в то же время - нётерова симметрия цепочки Тоды $\mathcal{E}_{\text {Тода }}$. Уравнение $u_{t_{1}}=\varphi_{1}$ гамильтоново относительно оператора $B_{1}=D_{x}^{-1} \kappa^{-1} ;$ уравнение $\mathcal{E}_{\mathrm{mKdV}}=\left\{\vartheta_{t_{1}}=\kappa D_{x}\left(\varphi_{1}\right)\right\}$, являющееся $r$-компонентным аналогом модифицированного уравнения КдФ, гамильтоново относительно $\widehat{B}_{1}=\kappa D_{x}$. Интеграл $w[\vartheta]$ задает преобразование Миуры между высшими модифицированными уравнениями КдФ и иерархией $\mathfrak{A}$ уравнения (1). Именно, условие (4) выполнено для подстановки $w=w[\vartheta]$ и второй гамильтоновой структуры $\hat{A}_{2}=-\beta D_{x}^{3}+D_{x} \circ w+w \cdot D_{x}$ для уравнения Кд $\Phi \mathcal{E}_{\mathrm{KdV}}$. Вследствие этого времена $t_{i}$ эволюции $u_{t_{i}}=\varphi_{i}$ согласованы со временами в иерархии $\mathfrak{A}$ уравнения $\mathcal{E}_{\mathrm{pKdV}}$ посредством указанного преобразования; все четыре уравнения $\mathcal{E}_{(\mathrm{p})(\mathrm{m}) \mathrm{KdV}}$, а также их высшие 
аналоги, допускают единый набор гамильтонианов $\mathcal{H}_{i}=\left[H_{i} d x\right]$.

УТВеРЖДЕНИЕ 5 [4]. Имеют место факторизации $R_{\text {Toda }}=\square \circ \ell_{s}$ u $R_{\mathrm{pKdV}}=\ell_{s} \circ$ $\square$ для операторов рекурсии $R_{\text {Toda }}: \varphi_{i} \mapsto \varphi_{i+1}$ и $R_{\mathrm{pKdV}}: \phi_{i} \mapsto \phi_{i+1}$. Каждый поток

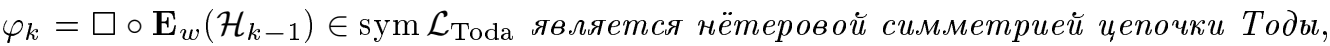
причем сечение $\varphi_{k}$ гамильтоново относительно гамильтоновой структуры $B_{1}=$ $\kappa^{-1} \cdot D_{x}^{-1}$ и гамильтониана $\mathcal{H}_{k-1}=\left[H_{k-1} d x\right]$ для $(k-1)$-го высшего уравнения $K \partial \Phi$.

УТВЕРЖДЕНИЕ 6. Симметрии $\varphi_{k}$ коммутируют.

ДокАЗАТЕЛЬСТво. Иерархия $\mathfrak{A}$ уравнения КдФ коммутативна и локальна по $s_{x}=$ $w$. Пусть $\varphi^{\prime}=\square\left(\phi^{\prime}(x, \boldsymbol{W})\right)$ и $\varphi^{\prime \prime}=\square\left(\phi^{\prime \prime}(x, \boldsymbol{W})\right)$, тогда скобка Якоби $\varphi^{\prime}$ и $\varphi^{\prime \prime}$ имеет вид $\left\{\varphi^{\prime}, \varphi^{\prime \prime}\right\}=\square\left(\phi_{\{1,2\}}(x, \boldsymbol{W})\right)$, где (приведенная ниже скобка введена в работе [1] применительно к скалярному случаю)

$$
\phi_{\{1,2\}}=\vartheta_{\varphi^{\prime}}\left(\phi^{\prime \prime}\right)-\vartheta_{\varphi^{\prime \prime}}\left(\phi^{\prime}\right)+\bar{D}_{x}\left(\phi^{\prime}\right) \phi^{\prime \prime}-\phi^{\prime} \bar{D}_{x}\left(\phi^{\prime \prime}\right) .
$$

С одной стороны, [Э$\left.Э_{\varphi_{k_{1}}}, Э_{\varphi_{k_{2}}}\right](w)=\left\{\Phi_{k_{1}}, \Phi_{k_{2}}\right\}=0 ;$ с другой стороны, справедливы равенства $\varphi_{k_{i}}=\square\left(\phi_{k_{i-1}}\right)$ и $\vartheta_{\left\{\varphi_{k_{1}}, \varphi_{k_{2}}\right\}}(w)=\hat{A}_{2}\left(\phi_{\left\{k_{1}, k_{2}\right\}}\right)$. Очевидно, гамильтонов оператор $\hat{A}_{2}$ для КдФ инъективен. Отсюда получаем, что $\phi_{\left\{k_{1}, k_{2}\right\}}=0$, и потому $\left\{\varphi_{k_{1}}\right.$, $\left.\varphi_{k_{2}}\right\}=\square(0)=0$. Это же рассуждение показывает, что оператор $R_{\text {Tода }}$ является рекурсией для $i$-го уравнения $u_{t_{i}}=\varphi_{i}$ в коммутативной подалгебре симметрий $\mathfrak{B}$ при всех $i \geqslant 0$.

\section{3. ИЕРАРХИЯ УРАВНЕНИЯ БУССИНЕСКА}

Первая гамильтонова структура для уравнения Буссинеска (2) есть $\hat{A}_{1}=\left(\begin{array}{cc}0 & D_{x} \\ D_{x} & 0\end{array}\right)$. Зададим потенциалы $u, v$ формулами $U=v_{x}, V=u_{x}$; переменные $u, v$ удовлетворяют потенциальному уравнению Буссинеска (2). В дальнейшем будут использоваться обозначения $\boldsymbol{u}={ }^{t}(u, v)$ и $\boldsymbol{U}={ }^{t}(U, V)$. Лагранжиан объемлюшего волнового уравнения $\mathcal{E}=\left\{v_{x y}=0, u_{x y}=0\right\}$ с плотностью $L_{\text {Bous }}=-u_{x} v_{y} / 2-v_{x} u_{y} / 2$, получается непосредственно. Одно из преобразований Миуры для уравнения (2) задано формулами [7]

$$
U=a^{2}+a b+b^{2}+2 a_{x}+b_{x}, \quad V=-2 a\left(b(a+b)+b_{x}\right)-D_{x}\left(b_{x}+a b+b^{2}\right) .
$$

Непотенциальное модифицированное уравнение Буссинеска таково:

$$
a_{t}=\frac{1}{3} D_{x}\left(a^{2}-2 a b-2 b^{2}-a_{x}-2 b_{x}\right), \quad b_{t}=\frac{1}{3} D_{x}\left(-2 a^{2}-2 a b+b^{2}+2 a_{x}+b_{x}\right) .
$$

Это уравнение гамильтоново относительно оператора $\widehat{B}_{1}=-\frac{1}{3}\left(\begin{array}{rr}2 & -1 \\ -1 & 2\end{array}\right) \cdot D_{x}$ и оператора Казимира $\mathcal{H}_{0}=[V d x]$. Обратим внимание на то, что $\widehat{B}_{1}$ содержит матрицу Картана $K_{\mathfrak{s} \mathfrak{l}_{3}}$ алгебры $\mathfrak{s l}_{3}(\mathbb{C})$, см. [6]. Отметим также, что вторая серия потоков для уравнения (2) начинается с оператора Казимира $\overline{\mathcal{H}}_{0}=\int[U d x]$; ясно также, что интегральные условия

$$
[U d x]=\int_{-\infty}^{+\infty} v_{x} d x=\text { const }, \quad[V d x]=\int_{-\infty}^{+\infty} u_{x} d x=\text { const }
$$


используемые в методе обратной задачи, имеют естественную природу. Заметим еще, что гамильтонианы $\mathcal{H}_{0}$ и $\overline{\mathcal{H}}_{0}$ порождают ненулевые потоки симметрии на уравнениях (2), а именно, сдвиги потенциальных переменных $u$ и $v$. Введем потенциалы $\alpha$ и $\beta$ такие, что $a=\left(2 \alpha_{x}-\beta_{x}\right) / 3, \quad b=\left(-\alpha_{x}+2 \beta_{x}\right) / 3$; положим $\boldsymbol{\alpha}={ }^{t}(\alpha, \beta)$ и $\boldsymbol{a}={ }^{t}(a, b)$. Гамильтоновы потоки $\boldsymbol{u}_{t_{i-1}}=\phi_{i-1}$ отображаются в потоки $\boldsymbol{\alpha}_{t_{i}}=\varphi_{i}=\square\left(\phi_{i-1}\right)$ под действием оператора

$$
\square=\left(\ell_{\boldsymbol{U}}^{\boldsymbol{a}}\right)^{*}=\left(\begin{array}{cc}
2 a+b-2 D_{x} & -4 a b-2 b^{2}-2 b_{x}+b D_{x} \\
a+2 b-D_{x} & -2 a^{2}-4 a b+2 D_{x} \circ a-D_{x}^{2}+(a+2 b) D_{x}
\end{array}\right) .
$$

Чтобы описать объемлюшее лагранжево уравнение $\mathcal{E}_{\mathrm{EL}}^{\prime}$, необходимо разрешить уравнения $\left\{\varphi_{i} \in \operatorname{sym} \mathcal{E}_{\mathrm{EL}}^{\prime}\right\}, i \geqslant 0$, относительно гамильтониана $H_{\mathrm{EL}}^{\prime}$. Решая первые два из них, находим $H_{\mathrm{EL}}^{\prime}=c_{1} \exp (\beta-\alpha)+c_{2} \exp (\alpha)+c_{3} \exp (-\beta)$, где $c_{1}, c_{2}, c_{3} \in \mathbb{R}$, и, таким образом, получаем двумерную цепочку Тоды. Функционалы $\boldsymbol{U}[\boldsymbol{a}]$ являются интегралами уравнения $\mathcal{E}_{\mathrm{EL}}^{\prime}$ (а значит, оно лиувиллевского типа), если $c_{2}=0$, и потому

$$
\alpha_{x y}=-c_{1} \exp (\beta-\alpha)-c_{3} \exp (-\beta), \quad \beta_{x y}=c_{1} \exp (\beta-\alpha)-2 c_{3} \exp (-\beta) .
$$

Это уравнение сводится к цепочке Тоды [6] $\boldsymbol{\alpha}_{x y}=\exp \left(K_{\mathfrak{s l}_{3}} \boldsymbol{\alpha}\right)$ заменой переменных. Вспомним, что $\phi_{i} \in \operatorname{im} \mathbf{E}_{\boldsymbol{U}}$ и $\square=\left(\ell_{\boldsymbol{U}}^{\boldsymbol{a}}\right)^{*}$. Следовательно, потоки $\varphi_{i}=\square\left(\phi_{i-1}\right)$ являются нётеровыми симметриями $\mathcal{E}_{\mathrm{EL}}^{\prime}$. В этом случае преобразование Миуры $\boldsymbol{U}=\boldsymbol{U}[\boldsymbol{a}]$ и разложение (4) задают естественную факторизацию второй гамильтоновой структуры [17]

$$
\hat{A}_{2}=\left(\begin{array}{cc}
-2 D_{x}^{3}+2 U D_{x}+U_{x} & 3 V D_{x}+2 V_{x} \\
3 V D_{x}+V_{x} & \frac{2}{3} D_{x}^{5}-\frac{5}{3}\left(U D_{x}^{3}+D_{x}^{3} \circ U\right)+ \\
& +U_{x x} D_{x}+D_{x} \circ U_{x x}+\frac{8}{3} U D_{x} \circ U
\end{array}\right)
$$

для уравнения Буссинеска (2). Начальные элементы иерархии Буссинеска и согласованной с ней последовательности потоков модифицированного уравнения Буссинеска, допускающие общий набор гамильтонианов $\mathcal{H}_{i}$, приведены в диаграмме $(5)$; плотности гамильтонианов таковы:

$$
\begin{aligned}
& H_{0}=V \\
& H_{1}=\frac{1}{2} V^{2}+\frac{1}{6} U_{x}^{2}+\frac{2}{9} U^{3}, \\
& H_{2}=\frac{1}{12} U_{x x} V_{x x}-\frac{5}{12} U U_{x x} V-\frac{5}{16} U_{x}^{2} V+\frac{5}{36} U^{3} V+\frac{5}{48} V^{3}, \ldots .
\end{aligned}
$$

Из утверждения 4 следует, что элементы $\varphi_{i}$ последовательности $\mathfrak{B}$ нётеровых потоков на цепочке Тоды (9), соответствуюшие модифицированному уравнению Буссинеска, попарно коммутируют и локальны по $\boldsymbol{a}$; доказательство этого факта аналогично приведенному выше для утверждения 6. Отметим, что явная формула (6), задающая скобку $\phi_{\left\{k_{i}, k_{j}\right\}}$, не используется. Кроме того, мы получаем факторизации $R_{\mathrm{pmBous}}=\square$ о $\hat{A}_{1}^{-1} \circ \square^{*} \circ \ell_{\boldsymbol{a}}^{\boldsymbol{\alpha}}$ и $R_{\mathrm{mBous}}=\ell_{\boldsymbol{a}}^{\boldsymbol{\alpha}} \circ \square$ ० $\hat{A}_{1}^{-1} \circ \square^{*}$ операторов рекурсии $R_{\mathrm{pmBous}}=R_{\mathrm{mBous}}^{*}$, размножаюших высшие модифицированные уравнения Буссинеска (8). Согласно утверждению 2 рекурсии для иерархий $\mathfrak{A}$ и $\mathfrak{B}$ слабо нелокальны. 
Благодарности. Автор благодарен А. М. Вербовецкому, Б. А. Дубровину, И. С. Красильщику и М. В. Павлову за полезные обсуждения, а также глубоко признателен В. В. Соколову и рецензенту за важные замечания и конструктивную критику. Автор выражает благодарность оргкомитету конференции "Нелинейная физика. Теория и эксперимент. III" за поддержку. Работа выполнена при частичной поддержке гранта INTAS YS 2001/2-33 и гранта университета Лечче № 650 CP/D.

\section{Список литературы}

[1] А. В. Жибер, В. В. Соколов. УМН. 2001. Т. 56. № 1. С. 63-106.

[2] F. Magri. J. Math. Phys. 1978. V. 19. № 5. P. 1156-1162.

[3] R. M. Miura. J. Math. Phys. 1968. V. 9. P. 1202-1204.

[4] A. V. Kiselev, A. V. Ovchinnikov. J. Dynam. Control Systems. 2004. V. 10. № 3. P. 431-451.

[5] А. Н. Лезнов, В. Г. Смирнов, А. Б. Шабат. ТМФ. 1982. Т. 51. № 1. С. 10-21.

[6] A. P. Fordy, J. Gibbons. J. Math. Phys. 1980. V. 21. № 10. P. 2508-2510; 1981. V. 22. № 6. P. $1170-1175$.

[7] М. В. Павлов. Фунд. прикл матем. 2004. Т. 10. № 1. С. 175-182.

[8] Y. Nutku, M. V. Pavlov. J. Math. Phys. 2002. V. 43. № 3. P. 1441-1459.

[9] А. В. Бочаров, А. М. Вербовецкий, А. М. Виноградов и др. Симметрии и законы сохранения уравнений математической физики. Ред. А. М. Виноградов и И. С. Красильщик. М.: Факториал, 1997.

[10] А. Г. Мешков. ТМФ. 1985. Т. 63. № 3. С. 323-332.

[11] G. Barnich, F. Brandt, M. Henneaux. Commun. Math. Phys. 1995. V. 174. P. 57-92; hep-th/9405109.

[12] A. V. Kiselev. Acta Appl. Math. 2004. V. 83. № 1-2. P. 175-182.

[13] C. S. Gardner. J. Math. Phys. 1971. V. 12. № 8. P. 1548-1551.

[14] P. Kersten, I. Krasil'shchik, A. Verbovetsky. J. Geom. Phys. 2004. V. 50. № 1-4. P. 273-302.

[15] А. Б. Шабат, Р. И. Ямилов. Экспоненциальные системы типа І и матрицы Картана. Препринт. Уфа: Башкир. филиал АН СССР, 1981.

[16] Д. К. Демской, С. Я. Стариев. Фунд. прикл. матем. 2004. Т. 10. № 1. С. 29-37.

[17] П. Олвер. Приложения групп Ли к дифференциальным уравнениям. М.: Мир, 1989.

[18] E. Getzler. Duke Math. J. 2002. V. 111. P. 535-560. 